\title{
Detection of new Yr1-virulences in Puccinia striiformis f. sp. tritici population and its sources of resistance in advance wheat lines and released cultivars
}

\author{
O.P. GANGWAR ${ }^{1}$, SUBODH KUMAR ${ }^{1}$, S.C. BHARDWAJ ${ }^{1 *}$, PRAMOD PRASAD $^{1}$, HANIF KHAN ${ }^{1}$, SIDDANNA SAVADI $^{1}$ \\ and S.K. SHARMA ${ }^{2}$ \\ ${ }^{1}$ Regional Station, ICAR-Indian Institute of Wheat and Barley Research, Flowerdale, Shimla 171 002, Himachal Pradesh, India \\ ${ }^{2}$ Regional Research Station, Mountain Agricultural Research \& Extension Centre, CSK Himachal Pradesh Krishi Vishvavidyalaya, \\ Sangla 172 106, Himachal Pradesh, India
}

Received: 6 June 2017/ Accepted: 18 August 2017/ Published online: 31 August 2017 (c) Indian Phytopathological Society 2017

\begin{abstract}
Five hundred and eight yellow rust (YR) samples were pathotyped from Northern states of India during 2014-15 and 2015-16 cropping seasons. The virulence of these samples was tested on 16 wheat differentials and 6 supplementary varieties. Eleven known pathotypes were identified from the analysis of 508 YR samples during this period. Additionally, the infection types of three samples on differentials, collected from US Nagar (Uttarakhand), Kangra and Kinnaur (Himachal Pradesh) districts, were dissimilar to the known pathotypes in India. Subsequently, three isolations were taken from each sample, established separately on susceptible check (A-9-30-1) and each isolation was used to inoculate differentials. All the isolations taken from single sample, showed similar reaction types on differentials. It proved the presence of new pathotype. The new pathotypes (virulences) were designated as 111S68, 79S4 and 79S68. Rust resistance genes Yr2 (Heines VII), Yr5, Yr9, Yr10, Yr15, Yr24, YrA were found effective to all these new pathotypes, identified in this study. Evaluation of 135 advance lines and released varieties of bread, durum, dicoccum wheat and triticale indicated that new pathotypes (111S68, 79S4, and 79S68) were less virulent than known pts. $47 \mathrm{~S} 103$ (T) and 47S102 (K) virulent to Yr1. More than 70 per cent evaluated wheat and triticale material was found resistant to moderately resistant to all Yr1-virulences. New pathotypes, identified from indigenous wheat, would aid in understanding the evolution mechanism in Yr1-virulences of yellow rust pathogen in India for better management of yellow rust.
\end{abstract}

Keywords: Evolution, pathotype, resistance, stripe rust, wheat

Wheat is the second most important cereal and food crop of India in terms of harvested area, production and consumption. India is the second largest producer (93.50 million tonnes during 2015-16) and consumer (88.55 million tonnes in 2015) of wheat in the world. Wheat production is mainly confined to the Indo-Gangetic plains region and three northern states, namely Uttar Pradesh (35.53\%), Punjab (18.96\%) and Haryana (13.39\%), supply 67 per cent of India's total wheat output (Tripathi and Mishra, 2017). Total demand of wheat in 2025 is forecasted to be in the range of 91.4-101.7 million tons (Ganesh-Kumar et al., 2012). The current major challenges facing future wheat production in India are increasing heat stress, dwindling water supplies for irrigation and ever-growing threat of new virulence of diseases such as wheat rusts and leaf blight (Joshi et al., 2007). Although many diseases and pests are known to reduce grain yield potential and quality of wheat, the three rusts fungi namely, Puccinia striiformis f. sp. tritici (Pst, Yellow/stripe rust), Puccinia triticina (Pt, Brown/leaf rust) and Puccinia graminis f. sp. tritici (Pgt, Black/stem rust) have historically caused major crop losses because

${ }^{*}$ Corresponding author: scbfdl@ hotmail.com of their widespread prevalence and airborne. Despite the widespread use of resistant cultivars and fungicides, rusts continue to remain a big challenge in India, bringing back unpleasant memories of pre-green revolution era. This group of diseases continue to remain economically important despite the widespread use of host resistance and fungicides (Singh et al., 2016). Yellow rust (YR) can cause 100 per cent yield losses if infection occurs very early and the disease continues developing during the growing season. In most wheat-producing areas, yield losses caused by stripe rust have ranged from 10 to 70 per cent depending on susceptibility of the cultivar, earliness of the initial infection, rate of disease development, and duration of disease (Chen, 2005). YR is more common in cooler areas of Northern India and Nilgiri hills in Southern India. It appeared in epidemic form in the plains of Jammu and Kashmir, foothills of Punjab and Himachal Pradesh and tarai region of Uttarakhand during 2010-11 crop season, but timely action on controlling its spread through fungicide saved the crop from major damage (Bhardwaj et al., 2012; Sharma and Saharan, 2011). Occurrence of yellow rust in high severity during the last decade was due to 
evolution of more virulent pathotypes that were able to overcome widely used sources of resistance in wheat (Prashar et al., 2007). Virulence information on Pst pathotypes is important to implement effective disease management with resistant cultivars (Sharma-Poudyal et al., 2013). The success of any breeding program to produce rust resistant cultivars depends on knowledge of virulences of a rust pathogen and their infection types on wheat. In this paper, detailed information about newly emerged Pst pathotypes virulent to Yr1 rust resistance gene and sources of resistance in advance wheat material and released varieties are discussed.

\section{MATERIALS AND METHODS}

\section{Yellow rust samples collection, analysis and determination of pathotypes}

Yellow rust leaf samples collected from infected wheat plants were established on 7-8 day old seedlings of susceptible line A-9-30-1. Fresh uredosporic growth appeared on A-9-30-1 within 15 days after inoculation (DAI). Inoculum (uredospores) was collected at $20^{\text {th }} \mathrm{DAI}$ from individual sample on a non-hygroscopic butter paper and was used for inoculating the sets of wheat differentials.

Pathotypes were identified based on infection types (ITs) produced on a group of 16 wheat differentials divided into three sub groups. The first sub group named as set-A (composed of selected world differentials) and the second sub group, designated as set-B included Indian and selected European differentials (Table 1). Set0 , third sub group consisted of Indian wheat cultivars developed recently along with two susceptible checks of wheat and barley which provided supplementary information useful for identification of different pathotypes. The seedlings of stripe rust differentials were grown in aluminium bread pans $(29 \mathrm{~cm}$ long $\times 12 \mathrm{~cm}$ wide $\times 7 \mathrm{~cm}$ deep size) in a mixture of fine loam and farmyard manure (3:1) that was sterilized by autoclaving at $60^{\circ} \mathrm{C}$ for an hour. Thirty holes in three rows $(3 \mathrm{~cm}$ depth and $4 \mathrm{~cm}$ apart, 10 holes in each row) were made with the help of wooden markers and all the constituents of the group were sown in one bread pan. For each differential and elite line only 5-6 seeds were sown in one hole. The inoculation was done on 6-8 days old seedlings by scraping the uredospores from the infected leaf with lancet needle and smearing the scraped inoculum on differential lines. Thereafter, differential sets were sprayed with a fine mist of water and placed in dew chambers $(\mathrm{RH}>80 \%)$ at $12 \pm 2^{\circ} \mathrm{C}$ for $48 \mathrm{~h}$ with $12 \mathrm{~h}$ of daylight photoperiod. The inoculated material was then removed and put on benches in a greenhouse where temperature of $16 \pm 2{ }^{\circ} \mathrm{C}$, relative humidity of $60-80 \%$ and illumination of about 15,000 lux for $12-16 \mathrm{~h}$ photoperiod were maintained.

Infection types of Pst pathotypes on wheat differentials were scored at 20 days post- inoculation (DPI), following the method suggested by Mclntosh et al. (1995). Infection types were characterized as 0 (Immune) $=$ no visible uredia, (Highly Resistant $)=$ Necrotic flecks; N (Resistant) = Necrotic area without sporulation, 1 (Resistant) = Necrotic and chlorotic areas with restricted sporulation, 2 (Moderately resistant) = moderate sporulation with necrosis and chlorosis, 3 (Moderately susceptible) $=$ Sporulation with chlorosis, $3+/ 4$ (Susceptible) $=$ Abundant sporulation without chlorosis. Infection type 33+ was used when both 3 and $3+$ pustules occurred together. The experiment was conducted twice to confirm the reaction types. If a Pst isolate showed any difference in pathogenicity from the old pathotypes, then, isolation was taken, reaction types confirmed and new pathotype was designated as per the binary notation system (Nagarajan et al., 1983) with modification (Bhardwaj et al., 2012).

\section{Evaluation of Indian advance wheat lines}

A set 135 advance wheat lines (AVT I, 2016-17) and selected released varieties comprising of aestivum, durum, dicoccum wheat and triticale, were evaluated against the three new pathotypes (111S68, 79S68, 79S4) and two known Yr1-virulent pts. 47S1103 (T) and 47S102 (K). The list of 135 wheat lines/varieties and their response to Pst pathotypes is shown in Table 4.

For evaluating wheat elite lines, only 18 holes were made in one bread pan. Further, the elite lines were inoculated using a glass atomizer containing $10 \mathrm{mg}$ spores of a Pst pathotype suspended in $2 \mathrm{ml}$ light grade

Table 1. Set of differentials for the identification of Pst pathotypes on wheat in India

\begin{tabular}{|c|c|c|c|c|c|}
\hline $\begin{array}{l}\text { Set-A (Selected } \\
\text { Word differentials) }\end{array}$ & Yr-gene & $\begin{array}{l}\text { Set-B (Selected European } \\
\text { and Indian differentials) }\end{array}$ & Yr-gene & $\begin{array}{c}\text { Supplemental } \\
\text { differential cultivars }\end{array}$ & Yr-gene \\
\hline Chinese166 & 1 & Hybrid46 & $3 b, 4 b$ & WH147 & - \\
\hline Lee & $7,22,23$ & HeinesVII & $2,+$ & Bilara 2 (Barley) & - \\
\hline Heines Kolben & 2,6 & Compair & 8,19 & PBW343 & 9,27 \\
\hline Vilmorin23 & $3 a, 4 a,+$ & TSA & $5,+$ & HS240 & $9,+$ \\
\hline Moro & 10 & Tc*6/Lr26 & $9,+$ & Anza & $A$ \\
\hline Strubes Dickkopf & 2, 3a\#, 4a\#, 25 & Sonalika (SK) & 2, $A,+$ & A-9-30-1 & - \\
\hline Suwon92 × Omar & Su & Kalyansona (KS) & $2,+$ & & \\
\hline Riebesel47/51 & $2,9,+$ & YR24/Yr26 & $24 / 26$ & & \\
\hline
\end{tabular}

- = no resistance gene known, + additional resistance genes known, \# status of these genes not yet known but continue to be mentioned in the literature 
Table 2. Distinguishing virulence of new and known Pst pathotypes on wheat differentials

\begin{tabular}{|c|c|c|c|c|c|c|}
\hline \multirow[t]{2}{*}{ Wheat differentials } & \multirow[t]{2}{*}{ Yr-gene } & \multicolumn{5}{|c|}{ New and known Pst Pathotypes } \\
\hline & & $47 \mathrm{~S} 102(\mathrm{~K})$ & 111S68* & $79 S 68^{*}$ & $79 S 4^{*}$ & $47 \mathrm{~S} 103(\mathrm{~T})$ \\
\hline Strubes Dickkopf & $2,3 a, 4 a, 25$ & $\mathrm{~V}$ & $\mathrm{~V}$ & $\mathrm{Av}$ & $\mathrm{Av}$ & $\mathrm{V}$ \\
\hline Suwon92 $\times$ Omar & $\mathrm{Su}$ & $A v$ & $\mathrm{~V}$ & $\mathrm{~V}$ & $\mathrm{~V}$ & $A v$ \\
\hline Hybrid46 & $3 b, 4 b$ & $A v$ & $A v$ & Av & Av & V \\
\hline HeinesVII & $2,+$ & $\mathrm{V}$ & $A v$ & $A v$ & $A v$ & $\mathrm{~V}$ \\
\hline Sonalika (SK) & $2, A,+$ & V & $A v$ & $A v$ & $A v$ & V \\
\hline Kalyansona (KS) & $2,+$ & V & $\mathrm{V}$ & $\mathrm{V}$ & $A v$ & $\mathrm{~V}$ \\
\hline Anza & $A$ & $\mathrm{~V}$ & $\mathrm{Av}$ & $A v$ & $A v$ & $\mathrm{~V}$ \\
\hline
\end{tabular}

* New Pst pathotypes identified in this study, Av-avirulent (0; , , , ;1), V-virulent (2+, 3-, 3+) based on 0-4 scale as described by Mclntosh et al. (1995)

Table 3. Avirulence /virulence structure of new pathotypes

\begin{tabular}{|c|c|c|c|}
\hline Pathotypes & $\begin{array}{l}\text { Location and year of } \\
\text { detection }\end{array}$ & Avirulence & Virulence \\
\hline $111 S 68$ & $\begin{array}{l}\text { US Nagar (Uttarakhand), } \\
2015\end{array}$ & $\begin{array}{l}\text { Yr2 (Heines VII), Yr3b, Yr4b } \\
\text { (Hybrid46), Yr5 (TSA), Yr9 } \\
\text { (Tc*6/Lr26), Yr2+Yr9 (Riebesel47/51), } \\
\text { Yr10 (Moro), Yr24 (or Yr26), YrA } \\
\text { (Sonalika) }\end{array}$ & $\begin{array}{l}\text { Yr1 (Chinese166), Yr2 (Kalyansona), Yr3a, } \\
\text { Yr4a (Vilmorin23), Yr2, Yr6 (Heines } \\
\text { Kolben), Yr8, Yr19 (Compair), Yr7, Yr22, } \\
\text { Yr23 (Lee), Yr2, Yr3a, Yr4a, Yr25 (Strubes } \\
\text { Dickkopf), YrSu (Suwon92 × Omar) }\end{array}$ \\
\hline $79 S 4$ & $\begin{array}{l}\text { Kangra, (Himachal } \\
\text { Pradesh), } 2016\end{array}$ & $\begin{array}{l}\text { Yr2 (Heines VII), Yr3b, Yr4b (Hybrid46), } \\
\text { Yr2, Yr3a, Yr4a, Yr25 (Strubes Dickkopf), } \\
\text { Yr2 (Kalyansona), Yr5 (TSA), Yr9 (Tc*6/ } \\
\text { Lr26), Yr2+Yr9 (Riebesel47/51), Yr10 } \\
\text { (Moro), Yr24 (or Yr26), YrA (Sonalika) }\end{array}$ & $\begin{array}{l}\text { Yr1 (Chinese166), Yr3a, Yr4a (Vilmorin23), } \\
\text { Yr2, Yr6 (Heines Kolben), Yr8, Yr19 } \\
\text { (Compair), Yr7, Yr22, Yr23 (Lee), YrSu } \\
\text { (Suwon92 × Omar) }\end{array}$ \\
\hline $79 S 68$ & $\begin{array}{l}\text { Kinnaur, (Himachal } \\
\text { Pradesh), } 2016\end{array}$ & $\begin{array}{l}\text { Yr2 (Heines VII), Yr3b, Yr4b (Hybrid46), } \\
\text { Yr2, Yr3a, Yr4a, Yr25 (Strubes Dickkopf), } \\
\text { Yr5 (TSA), Yr9 (Tc*6/Lr26), Yr2+Yr9 } \\
\text { (Riebesel } 47 / 51), Y r 10 \text { (Moro), Yr24 } \\
\text { (or Yr26), YrA (Sonalika) }\end{array}$ & $\begin{array}{l}\text { Yr1 (Chinese166), Yr2 (Kalyansona), Yr3a, } \\
\text { Yr4a (Vilmorin23), Yr2, Yr6 (Heines } \\
\text { Kolben), Yr8, Yr19 (Compair), Yr7, Yr22, } \\
\text { Yr23 (Lee), YrSu (Suwon92 × Omar) }\end{array}$ \\
\hline
\end{tabular}

mineral oil (Soltrol 170®) (Chevron Phillips Chemicals Asia Pvt. Ltd., Singapore). After inoculation, the oil was allowed to evaporate for $10 \mathrm{~min}$. Remaining study was conducted as described above for differentials.

\section{RESULTS}

\section{Phenotypic characterization of new pathotypes}

During 2014-15 cropping season, 295 yellow rust samples were pathotyped on wheat differentials. A sample (Pst-254/36.2015) collected from infected leaves of local wheat variety grown at farmer's field located in US Nagar district of Uttarakhand, showed reaction types different from known Yr1-virulences. Isolations were taken, one each from chinese166, Strubes Dickkopf and Suwon92 $\times$ Omar and were multiplied on susceptible check (A-9-30-1). Subsequently, each isolation was used separately to inoculate the sets of differentials and compared with closely related Yr1-virulences 47S102 and 47S103. The test isolations showed similar kind of reactions but different from known pathotypes (Table 2). The test isolation possessed the combined virulence for Strubes Dickkopf and Suwon92 $\times$ Omar. This new Yr1 virulence was designated as 111S68. The avirulence/ virulence formula is described in Table 3.
In total, 213 YR samples were characterised on wheat differentials during 2015-16 cropping season. A sample (Pst-162/10.2016) was collected from wheat leaves with uredospores from Kangra district of Himachal Pradesh, showed reactions on differentials which were distinct from known pathotypes virulent to $Y r 1$. Isolations taken from differentials, like the earlier one was first established on susceptible host, and then compared with closely related known pts. $47 \mathrm{~S} 102$ and 47S103. All Isolations taken from YR sample (Pst-162/10.2016) showed similar reactions on differentials but distinct from the known ones. It proved that this was a new pathotype and designated as 79S4.

Similarly, another sample (Pst-01/10.2016) which was collected from Kinnaur district of Himachal Pradesh, possessed virulence to $Y r 1$ but different from known ones, was designated as 79568. The avirulence/virulence formula is described in Table 3.

New pt.111S68 is different to the known pt.47S103 (T) in having additional virulence for Suwon9 $\times$ Omar and lacking virulence for Hybrid46, Heines VII and Sonalika $(Y r A)$. This new Pst pathotype possesses combined virulence to Strubes Dickkopf and Suwon92 $\times$ Omar. The pts. 79568 and 7954 are distinguishable on Kalyansona (Yr2) as shown in Table 2. The former 
Table 4. Response of advance wheat lines (AVT I, 2016-17) and released varieties to new and known Yr-virulences of Puccinia striiformis f. sp. tritici

\begin{tabular}{|c|c|c|c|c|c|}
\hline $\begin{array}{l}\text { Advance wheat lines and } \\
\text { released varieties }\end{array}$ & 47S102 (K) & $79 S 68^{*}$ & $79 S 4^{*}$ & $111 S 68^{*}$ & $47 \mathrm{~S} 103(\mathrm{~T})$ \\
\hline AKDW2997-16(D)\# & MS & $\mathrm{R}$ & $\mathrm{R}$ & MS & $\mathrm{s}$ \\
\hline BRW3773 & $S$ & $R$ & $\mathrm{R}$ & MS & $S$ \\
\hline BRW3775 & MS & $\mathrm{R}$ & $\mathrm{R}$ & $\mathrm{R}$ & MS \\
\hline C306\# & $\mathrm{R}$ & MS & $\mathrm{R}$ & $\mathrm{R}$ & $S$ \\
\hline CG1023 & $s$ & MS & $\mathrm{R}$ & MR & $\mathrm{R}$ \\
\hline DBW14\# & $\mathrm{R}$ & $\mathrm{R}$ & $\mathrm{R}$ & MR & $\mathrm{R}$ \\
\hline DBW39\# & $R$ & $\mathrm{R}$ & $\mathrm{R}$ & $\mathrm{R}$ & $\mathrm{R}$ \\
\hline DBW71\# & $\mathrm{R}$ & $R$ & $\mathrm{R}$ & $\mathrm{R}$ & $\mathrm{R}$ \\
\hline DBW88\# & $\mathrm{R}$ & $R$ & $\mathrm{R}$ & $\mathrm{R}$ & $\mathrm{R}$ \\
\hline DBW90\# & $\mathrm{R}$ & $\mathrm{R}$ & $\mathrm{R}$ & $\mathrm{R}$ & $\mathrm{s}$ \\
\hline DBW110\# & $\mathrm{R}$ & $\mathrm{R}$ & $\mathrm{R}$ & $\mathrm{R}$ & MS \\
\hline DBW168 & S & $\mathrm{R}$ & $\mathrm{R}$ & $\mathrm{R}$ & $\mathrm{R}$ \\
\hline DBW173 & $\mathrm{R}$ & $\mathrm{R}$ & $\mathrm{R}$ & $\mathrm{R}$ & $\mathrm{R}$ \\
\hline DBW179 & $\mathrm{R}$ & $\mathrm{R}$ & $\mathrm{R}$ & $\mathrm{R}$ & $S$ \\
\hline DBW187 & S & $\mathrm{R}$ & $\mathrm{R}$ & $\mathrm{R}$ & MS \\
\hline DBW189 & $\mathrm{R}$ & MS & $\mathrm{R}$ & $\mathrm{R}$ & $\mathrm{R}$ \\
\hline DBW196 & S & $S$ & $\mathrm{R}$ & $\mathrm{R}$ & s \\
\hline DBW246 & $\mathrm{R}$ & $\mathrm{R}$ & $\mathrm{R}$ & $\mathrm{R}$ & $\mathrm{R}$ \\
\hline DBW247 & $S$ & MR & $\mathrm{R}$ & MS & s \\
\hline DBW248 & MR & $\mathrm{R}$ & $\mathrm{R}$ & $\mathrm{R}$ & $\mathrm{R}$ \\
\hline DBW249 & $\mathrm{R}$ & $\mathrm{R}$ & $\mathrm{R}$ & MS & MS \\
\hline DBW250 & $\mathrm{R}$ & $\mathrm{R}$ & $\mathrm{R}$ & MS & $S$ \\
\hline DBW251 & $\mathrm{R}$ & $\mathrm{R}$ & $\mathrm{R}$ & $\mathrm{R}$ & $s$ \\
\hline DDK1029 (Dic)\# & MS & $S$ & $\mathrm{R}$ & S & MS \\
\hline DDK1052 (Dic) & MS & MS & $\mathrm{R}$ & $\mathrm{R}$ & $\mathrm{R}$ \\
\hline DDK1053 (Dic) & $S$ & $S$ & $\mathrm{R}$ & $S$ & MS \\
\hline GW322\# & S & $R$ & $\mathrm{R}$ & MS & $S$ \\
\hline HD2733\# & $R$ & $R$ & $\mathrm{R}$ & $\mathrm{R}$ & $\mathrm{R}$ \\
\hline HD2828\# & $\mathrm{R}$ & $R$ & $\mathrm{R}$ & $\mathrm{R}$ & $S$ \\
\hline HD2967\# & $S$ & R & $\mathrm{R}$ & $\mathrm{R}$ & $S$ \\
\hline HD3043\# & $\mathrm{R}$ & $R$ & $\mathrm{R}$ & $\mathrm{R}$ & $\mathrm{R}$ \\
\hline HD3059\# & $\mathrm{R}$ & $R$ & $\mathrm{R}$ & $\mathrm{R}$ & MR \\
\hline HD3086\# & S & $R$ & $\mathrm{R}$ & $\mathrm{R}$ & $S$ \\
\hline HD3171\# & $\mathrm{s}$ & $R$ & $\mathrm{R}$ & $\mathrm{R}$ & $\mathrm{R}$ \\
\hline HD3219 & MS & $R$ & s & $s$ & MS \\
\hline HD3226 & MS & R & $\mathrm{R}$ & $\mathrm{R}$ & $\mathrm{R}$ \\
\hline HD3237 & $S$ & R & $\mathrm{R}$ & MS & MS \\
\hline HD3271 & $\mathrm{R}$ & $R$ & $\mathrm{R}$ & $\mathrm{R}$ & $\mathrm{R}$ \\
\hline HD3272 & MR & $R$ & $\mathrm{R}$ & $\mathrm{R}$ & S \\
\hline HI1612 & $\mathrm{R}$ & $R$ & $\mathrm{R}$ & $\mathrm{R}$ & $s$ \\
\hline HI1617 & $\mathrm{R}$ & $R$ & $\mathrm{R}$ & $\mathrm{R}$ & $\mathrm{R}$ \\
\hline HI1619 & $S$ & $R$ & $\mathrm{R}$ & $\mathrm{R}$ & $s$ \\
\hline HI1620 & S & $\mathrm{R}$ & $\mathrm{R}$ & $\mathrm{R}$ & S \\
\hline HI1621 & s & MS & MS & MS & S \\
\hline HI8627 (D) \# & $R$ & $\mathrm{R}$ & $\mathrm{R}$ & $\mathrm{R}$ & $\mathrm{R}$ \\
\hline HI8777 (D) & S & $R$ & $\mathrm{R}$ & $\mathrm{R}$ & $\mathrm{R}$ \\
\hline HI8791(D) & $R$ & R & $\mathrm{R}$ & $\mathrm{R}$ & $\mathrm{R}$ \\
\hline \multirow[t]{2}{*}{ HP1963 } & s & $\mathrm{R}$ & $\mathrm{R}$ & $\mathrm{R}$ & $\mathrm{s}$ \\
\hline & & & & & Contd. \\
\hline
\end{tabular}




\begin{tabular}{|c|c|c|c|c|c|}
\hline HPW251\# & $\mathrm{R}$ & $\mathrm{R}$ & $\mathrm{R}$ & $\mathrm{R}$ & $\mathrm{R}$ \\
\hline HPW439 & $\mathrm{R}$ & $\mathrm{R}$ & $\mathrm{R}$ & $\mathrm{R}$ & s \\
\hline HPW440 & $\mathrm{R}$ & MR & $\mathrm{R}$ & $\mathrm{R}$ & MS \\
\hline HPW448 & $\mathrm{R}$ & $\mathrm{R}$ & $\mathrm{R}$ & $\mathrm{R}$ & $\mathrm{R}$ \\
\hline HPW449 & $\mathrm{R}$ & R & $\mathrm{R}$ & $\mathrm{R}$ & $\mathrm{R}$ \\
\hline HS375\# & $\mathrm{R}$ & $\mathrm{R}$ & $\mathrm{R}$ & $\mathrm{R}$ & $\mathrm{R}$ \\
\hline HS490\# & $\mathrm{R}$ & $\mathrm{R}$ & $\mathrm{R}$ & $\mathrm{R}$ & $\mathrm{R}$ \\
\hline HS507\# & $\mathrm{R}$ & R & R & $\mathrm{R}$ & $\mathrm{R}$ \\
\hline HS542\# & $\mathrm{R}$ & R & $\mathrm{R}$ & $\mathrm{R}$ & MS \\
\hline HS611 & $S$ & $R$ & $\mathrm{R}$ & MS & $S$ \\
\hline HS629 & MS & R & $\mathrm{R}$ & $S$ & $\mathrm{R}$ \\
\hline HS630 & $S$ & $\mathrm{R}$ & $\mathrm{R}$ & MS & $\mathrm{s}$ \\
\hline HS643 & s & MS & $\mathrm{R}$ & $\mathrm{s}$ & $\mathrm{s}$ \\
\hline HS644 & R & $\mathrm{R}$ & R & $\mathrm{R}$ & $\mathrm{R}$ \\
\hline HS645 & R & R & $\mathrm{R}$ & $\mathrm{R}$ & $\mathrm{R}$ \\
\hline HS646 & $\mathrm{R}$ & R & R & $\mathrm{R}$ & $\mathrm{R}$ \\
\hline HS647 & $\mathrm{R}$ & $R$ & $\mathrm{R}$ & $\mathrm{R}$ & $\mathrm{R}$ \\
\hline HS648 & MS & $R$ & $\mathrm{R}$ & $\mathrm{R}$ & S \\
\hline HW1098\# & MS & $S$ & $\mathrm{R}$ & MS & MS \\
\hline HW2044\# & $\mathrm{R}$ & R & R & $\mathrm{R}$ & $\mathrm{R}$ \\
\hline HW5216\# & $\mathrm{R}$ & $R$ & $\mathrm{R}$ & $\mathrm{R}$ & $\mathrm{R}$ \\
\hline K0307\# & $S$ & $\mathrm{R}$ & R & MS & s \\
\hline K1006\# & S & $\mathrm{R}$ & $\mathrm{R}$ & S & s \\
\hline K1317\# & $\mathrm{s}$ & $\mathrm{R}$ & $\mathrm{R}$ & $\mathrm{s}$ & MS \\
\hline K8027\# & s & $\mathrm{R}$ & $\mathrm{R}$ & $\mathrm{R}$ & MS \\
\hline Kharchia 65\# & s & $\mathrm{s}$ & S & s & s \\
\hline KRL19\# & S & $S$ & $\mathrm{R}$ & $S$ & S \\
\hline KRL210\# & S & $R$ & $\mathrm{R}$ & $\mathrm{R}$ & S \\
\hline KRL370 & S & R & R & MS & S \\
\hline KRL377 & MS & $\mathrm{R}$ & $\mathrm{R}$ & $\mathrm{R}$ & MS \\
\hline KRL384 & $\mathrm{s}$ & $\mathrm{R}$ & $\mathrm{R}$ & $\mathrm{R}$ & $\mathrm{R}$ \\
\hline KRL386 & $S$ & R & $\mathrm{R}$ & MS & $S$ \\
\hline MACS4028 (D) & S & $S$ & MS & $S$ & $\mathrm{~s}$ \\
\hline MACS5047 & $S$ & MS & $\mathrm{R}$ & MS & MS \\
\hline MACS5049 & MR & $\mathrm{R}$ & R & MS & MS \\
\hline MACS6222\# & $\mathrm{R}$ & $\mathrm{R}$ & $\mathrm{R}$ & MS & MS \\
\hline MACS6478\# & MS & $\mathrm{R}$ & $\mathrm{R}$ & MS & MS \\
\hline MACS6677 & $S$ & R & R & $\mathrm{R}$ & $S$ \\
\hline MP1318 & $\mathrm{R}$ & R & $\mathrm{R}$ & $\mathrm{R}$ & $\mathrm{R}$ \\
\hline MP3288\# & MS & $\mathrm{R}$ & $\mathrm{R}$ & $\mathrm{R}$ & MS \\
\hline NI5439\# & $S$ & S & S & $S$ & $S$ \\
\hline NIAW1415\# & $\mathrm{R}$ & $\mathrm{R}$ & $\mathrm{R}$ & $\mathrm{R}$ & $\mathrm{R}$ \\
\hline PBW550\# & $\mathrm{R}$ & R & R & $\mathrm{R}$ & $\mathrm{R}$ \\
\hline PBW644\# & $\mathrm{R}$ & $\mathrm{R}$ & $\mathrm{R}$ & MS & $\mathrm{R}$ \\
\hline PBW750 & $\mathrm{R}$ & R & $\mathrm{R}$ & $\mathrm{R}$ & $\mathrm{R}$ \\
\hline PBW752 & $\mathrm{R}$ & $\mathrm{R}$ & $\mathrm{R}$ & $\mathrm{R}$ & $\mathrm{R}$ \\
\hline PBW757 & $\mathrm{R}$ & $\mathrm{R}$ & $\mathrm{R}$ & $\mathrm{R}$ & $\mathrm{R}$ \\
\hline PBW777 & $\mathrm{R}$ & $\mathrm{R}$ & $\mathrm{R}$ & $\mathrm{R}$ & $\mathrm{R}$ \\
\hline PBW778 & MS & MR & $\mathrm{R}$ & $\mathrm{R}$ & $\mathrm{s}$ \\
\hline PBW779 & $\mathrm{R}$ & $\mathrm{R}$ & $\mathrm{R}$ & $\mathrm{R}$ & $\mathrm{R}$ \\
\hline PBW780 & $\mathrm{R}$ & $\mathrm{R}$ & $\mathrm{R}$ & $\mathrm{R}$ & $\mathrm{R}$ \\
\hline TL2942\# & $\mathrm{R}$ & $\mathrm{R}$ & $\mathrm{R}$ & $\mathrm{R}$ & $\mathrm{R}$ \\
\hline
\end{tabular}




\begin{tabular}{|c|c|c|c|c|c|}
\hline TL2969\# & $\mathrm{R}$ & $\mathrm{R}$ & $\mathrm{R}$ & $\mathrm{R}$ & $\mathrm{R}$ \\
\hline TL3011 & $\mathrm{R}$ & $\mathrm{R}$ & $\mathrm{R}$ & $\mathrm{R}$ & $\mathrm{R}$ \\
\hline TL3012 & R & R & $\mathrm{R}$ & $\mathrm{R}$ & $\mathrm{R}$ \\
\hline TL3013 & $\mathrm{R}$ & $\mathrm{R}$ & $\mathrm{R}$ & $\mathrm{R}$ & $\mathrm{R}$ \\
\hline TL3014 & R & R & $\mathrm{R}$ & $\mathrm{R}$ & $\mathrm{R}$ \\
\hline TL3015 & $\mathrm{R}$ & $R$ & $\mathrm{R}$ & $\mathrm{R}$ & $\mathrm{R}$ \\
\hline UAS304\# & S & MS & $S$ & S & S \\
\hline UAS375 & $\mathrm{R}$ & $\mathrm{R}$ & $\mathrm{R}$ & $\mathrm{R}$ & $\mathrm{R}$ \\
\hline UAS384 & $S$ & R & $\mathrm{R}$ & $S$ & $\mathrm{R}$ \\
\hline UAS385 & S & $\mathrm{R}$ & $\mathrm{R}$ & MS & $S$ \\
\hline UAS387 & $\mathrm{R}$ & R & $\mathrm{R}$ & $\mathrm{R}$ & $\mathrm{R}$ \\
\hline UAS446\# & MS & S & $\mathrm{R}$ & MS & MS \\
\hline UAS462 (D) & $\mathrm{R}$ & MS & $\mathrm{s}$ & $S$ & $\mathrm{~s}$ \\
\hline UP2942 & $\mathrm{R}$ & $\mathrm{R}$ & $\mathrm{R}$ & $\mathrm{R}$ & $\mathrm{R}$ \\
\hline UP2992 & MS & $\mathrm{R}$ & $\mathrm{R}$ & $\mathrm{R}$ & MS \\
\hline UP2993 & $\mathrm{R}$ & $\mathrm{R}$ & $\mathrm{R}$ & $\mathrm{R}$ & $\mathrm{R}$ \\
\hline VL829\# & $\mathrm{R}$ & $\mathrm{R}$ & $\mathrm{R}$ & $\mathrm{R}$ & $\mathrm{R}$ \\
\hline VL892\# & $\mathrm{R}$ & R & $\mathrm{R}$ & $\mathrm{R}$ & $\mathrm{R}$ \\
\hline VL1011 & MS & $R$ & $\mathrm{R}$ & $\mathrm{R}$ & $\mathrm{R}$ \\
\hline VL1012 & $\mathrm{R}$ & $R$ & $\mathrm{R}$ & $\mathrm{R}$ & $\mathrm{R}$ \\
\hline VL1013 & $R$ & $R$ & $\mathrm{R}$ & $\mathrm{R}$ & $\mathrm{R}$ \\
\hline VL3013 & s & $\mathrm{R}$ & $\mathrm{R}$ & $\mathrm{R}$ & $\mathrm{R}$ \\
\hline VL3014 & S & $R$ & $\mathrm{R}$ & $\mathrm{R}$ & MS \\
\hline VL3015 & $\mathrm{R}$ & R & $\mathrm{R}$ & $\mathrm{R}$ & $\mathrm{R}$ \\
\hline VL4003 & $R$ & $R$ & $\mathrm{R}$ & $\mathrm{R}$ & $\mathrm{R}$ \\
\hline WH1021\# & R & $R$ & $\mathrm{R}$ & $\mathrm{R}$ & $\mathrm{R}$ \\
\hline WH1080\# & S & $R$ & $\mathrm{R}$ & MS & $S$ \\
\hline WH1105\# & $\mathrm{R}$ & R & $\mathrm{R}$ & $\mathrm{R}$ & MS \\
\hline WH1124\# & MS & $R$ & $\mathrm{R}$ & $\mathrm{R}$ & s \\
\hline WH1142\# & $\mathrm{R}$ & R & $\mathrm{R}$ & $\mathrm{R}$ & $\mathrm{R}$ \\
\hline WH1202 & R & R & $\mathrm{R}$ & $\mathrm{R}$ & $\mathrm{R}$ \\
\hline WH1232 & R & R & $\mathrm{R}$ & $\mathrm{R}$ & $S$ \\
\hline WH1233 & $\mathrm{R}$ & $R$ & $\mathrm{R}$ & $\mathrm{R}$ & $\mathrm{R}$ \\
\hline WH1316 & MS & S & $\mathrm{R}$ & $S$ & MS \\
\hline WR544\# & $s$ & $\mathrm{R}$ & $\mathrm{R}$ & MS & $\mathrm{s}$ \\
\hline
\end{tabular}

$\mathrm{D}=$ Durum wheat, $\mathrm{Dic}=$ Dicoccum wheat, ${ }^{*}$ New pathotypes, \# released varieties

pathotype is virulent on Kalyansona (Yr2), whereas, the latter one is avirulent.

\section{Evaluation of advance wheat lines and released varieties}

In total, 135 advance lines and released varieties of bread, durum, dicoccum wheat and Triticale were screened for rust resistance against new (79S68, 79S4 and 111S68) and known (47S102 and 47S103) pathotypes of yellow rust pathogen (Table 4). In this study, $53(39.3 \%)$ lines/varieties were found immune (IT: 0;, ; or ;-) and only two genotype, Kharchia65 and NI5439, highly susceptible (IT: $3+, 33+)$ to all pathotypes possessed virulence to Yr1. Among 135 advance lines/ released varieties, 92 were found resistant to moderately resistant (IT: 0; , ;, ; 1, 2-) to new pts. 79S68, 7954 and 111S68. Advance line DBW189 and a released variety PBW644 which were resistant to both known pathotypes (47S103 and 46S102), showed moderately susceptibility to new pts. $79 S 68$ and 111S68, respectively. On the other hand, BRW3775, DBW187, HD2967, HD3086, HI1619, HI1620, HI1963, HS648, K8027, KRL210, KRL377, MACS6677, MP3288, PBW778, UP2992, VL3014 and WH1124 were resistant to new pathotypes (79S68, 79S4 and 111S68) but found susceptible to moderately susceptible to known pathotypes ( $T$ and $\mathrm{K}$ ). In toto, 74 , $113,128,95$ and 68 advance lines/ released varieties were resistant, 17, 9, 2, 23 and 24 lines moderately susceptible, $41,10,5,15$ and 42 lines susceptible to pts. 47S102 (K), 79S68, 79S4, 111 S68 and 47S103 (T), respectively (Fig. 1). 


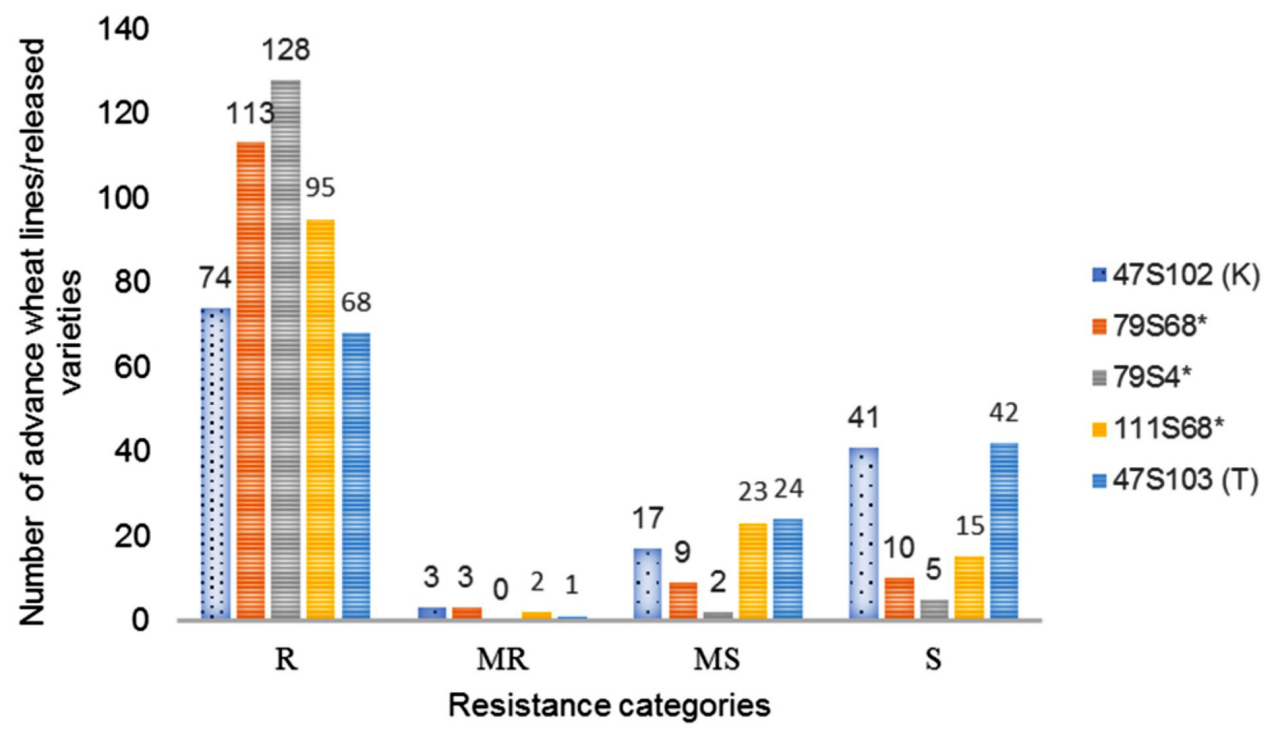

Fig. 1. Number of advance lines and released varieties of bread, durum, dicoccum wheat and Triticale in different resistance categories to new and known Yr1-virulent pathotypes of yellow rust pathogen

\section{DISCUSSION}

Deploying the rust resistance genes in wheat cultivars remains a sustainable approach to manage this devastating wheat rust diseases. However, new pathotypes/races evolved quickly rendering previously resistant cultivars susceptible (Wan and Chen, 2012; Bhardwaj et al., 2016). In India, first virulence to $Y r 1$ was detected in 1936 and designated as 31 (67S64). Subsequently, pt. 13 (67S8) in 1937, K (47S102) in 1982, T (47S103) in 1992, CII (15S64) in 1993 and 7 S0 in 2012, were identified and confirmed based on reaction types on wheat differentials (Nayar et al., 1997; Gangwar et al., 2016). In Indian Pst population, many virulences have been detected on $Y r 1$ even though there is no evidence of its use in wheat breeding programme. Such mutation, unrelated to host resistance genes, have been observed earlier and were attributed to their associations with better survival/adaptation of the pathogen. Authors of this study also felt that these mutations have provided yellow rust pathogen with an advantage. Mutation coupled with the directional selection, somatic and sexual recombination are considered to be mechanisms determining the genetic variability of Puccinia striiformis $\mathrm{f}$. $\mathrm{sp}$. tritici that result in the evolution of new aggressive races or pathotypes and breakdown of major resistance gene (Hovmoller et al., 2011; Jin, 2011; Park and Wellings, 2012; McDonald and Linde, 2002). In present study, three new Yr1 virulences of yellow rust pathogen which were identified in the year 2015 (pt. 111S68) and 2016 (79S4, $79568)$, also presumed to have evolved by the mechanism of mutation. The virulence spectra of these pathotypes is narrower than earlier known Yr1-virulences ( $T$ and $K$ ) as is evident from data on differentials and wheat genotypes. However, their geographical distribution is wider than previously identified Yr1-virulent pt. 15 S64 (CII) which is less virulent and confined to LehLadakh regions of Jammu and Kashmir (Bhardwaj et al., 2012). Although, more than 70 per cent wheat advance lines and released varieties retained resistance to these pathotypes but a significant portion of the evaluated lines were found susceptible to new pathotypes. New pathotypes have been added to national repository of pathotypes of rust pathogens at ICAR-IIWBR, Shimla, Himachal Pradesh.

\section{ACKNOWLEDGEMENTS}

The authors are thankful to the Director, ICAR-Indian Institute of Wheat and Barley Research, Karnal, Haryana, India for providing liberal support to undertake the survey work.

\section{REFERENCES}

Bhardwaj SC, Gangwar OP, Singh SB, Saharan MS and Sharma $S$ (2012). Rust situation and pathotypes of Puccinia species in Leh Ladakh in relation to recurrence of wheat rusts in India. Indian Phytopath. 65: 230-232.

Bhardwaj SC, Prasad P, Gangwar OP, Khan H and Kumar S (2016) Wheat rust research-then and now. Indian J. Agr. Sci. 86: 1231-1244.

Chen XM (2005). Epidemiology and control of stripe rust (Puccinia striiformis f. sp. tritici) on wheat. Can. J. Plant Pathol. 27: 314-337.

Ganesh-Kumar A, Rajesh Mehta HP, Sanjay K, Prasad KG and Gulati A (2012). Demand and supply of cereals in India. IFPRI Discussion Paper 01158, New Delhi: International Food Policy Research Institute.

Gangwar OP, Kumar S, Prasad P, Bhardwaj SC, Khan H and Verma $H(2016)$. Virulence pattern and emergence of new pathotypes in Puccinia striiformis f. sp. tritici during 201115 in India. Indian Phytopath. 69: 178-185.

Hovmoller MS, Sorensen CK, Walter S and Justesen AF (2011). Diversity of Puccinia striiformis on cereals and grasses. Annu. Rev. Phytopathol. 49: 197-217.

Jin Y (2011). Role of Berberis spp. as alternate hosts in generating new races of Puccinia graminis and $P$. striiformis. Euphytica 179: 105-108. 
Joshi AK, Mishra B, Chatrath R, Ferrara GO and Singh RP (2007) Wheat improvement in India: present status, emerging challenges and future prospects. Euphytica 157: 431-446.

McDonald BA and Linde C (2002). Pathogen population genetics, evolutionary potential and durable rsistance. Annu. Rev. Phytopathol. 40: 349-379.

McIntosh RA, Wellings CR and Park RF (1995). Wheat rusts: an atlas of resistance genes. CSIRO Publishing, Melbourne, pp. 199.

Nagarajan S, Nayar SK and Bahadur P (1983). The proposed brown rust of wheat (Puccinia recondita f. sp. tritici) virulence analysis system. Curr. Sci. 52: 413-416.

Nayar SK, Prashar M and Bhardwaj SC (1997). Manual of current techniques in wheat rust. Research Bulletin 2, Regional Station, Directorate of Wheat Research, Flowerdale Shimla, Himachal Pradesh, India, pp. 1-32.

Park RF and Wellings CR (2012). Somatic Hybridization in the Uredinales. Annu. Rev. Phytopathol. 50: 219-239.

Prashar M, Bhardwaj SC, Jain SK and Datta D (2007). Pathotypic evolution in Puccinia striiformis in India during 1995-2004. Aust. J. Agric. Res. 58: 602-604.
Sharma-Poudyal D, Chen XM, Wan AM, Zhan GM, Kang ZS, Cao SQ, Jin SL, Morgounov A, Akin B, Mert Z, Shah SJA, Bux $\mathrm{H}$, Ashraf M, Sharma RC, Madariaga R, Puri KD, Wellings C, Xi KQ, Wanyera R, Manninger K, Ganzález MI, Koyda M, Sanin S, Patzek LJ (2013). Virulence characterization of international collections of the wheat stripe rust pathogen, Puccinia striiformis f. sp. tritici. Plant Dis. 97: 379-386.

Sharma I and Saharan MS (2011). Status of wheat disease in India with a special reference to stripe rust. Plant Dis. Res. 26: 156.

Singh RP, Singh PK, Rutkoski J, Hodson DP, He X, Jorgensen LN, Hovmoller MS and Huerta-Espino J (2016). Disease impact on wheat yield potential and prospects of genetic control. Annu. Rev. Phytopathol. 54: 303-322.

Tripathi A and Mishra AK (2017). The wheat sector in India: production, policies and food security. In: The Eurasian Wheat Belt and Food Security (eds. Gomez y Paloma et al., 2017). Springer International Publishing, Switzerland, pp. 275-296.

Wan AM and Chen XM (2012) Virulence, frequency, and distribution of races of Puccinia striiformis f. sp. tritici and Puccinia striiformis $\mathrm{f}$. sp. hordei identified in the United States in 2008 and 2009. Plant Dis. 96: 67-74. 